\title{
Embryonic valproate exposure alters mesencephalic dopaminergic neurons distribution and septal dopaminergic gene expression in domestic chicks
}

$1 \quad$ Alice Adiletta $^{1 \dagger}$, Alessandra Pross ${ }^{1,2,3 \dagger}$, Nicolò Taricco $^{1}$, Paola Sgadò $^{*}$

$2{ }^{1}$ Center for Mind/Brain Sciences, University of Trento, Rovereto, Italy

$3 \quad{ }^{2}$ Lleida's Institute for Biomedical Research-Dr. Pifarre Foundation (IRBLleida), Spain

$4 \quad{ }^{3}$ Dept. Experimental Medicine, University of Lleida, Spain.

12 * Correspondence:

13 Paola Sgadò

14 paola.sgado@unitn.it

$15{ }^{\dagger}$ These authors have contributed equally to this work and share first authorship

17 Keywords: Autism Spectrum Disorder, valproic acid, brain development, dopamine, 18 midbrain, septum, domestic chicks. 


\section{Abstract}

20 In recent years, the role of the dopaminergic system in the regulation of social behavior is

21 being progressively outlined, and dysfunctions of the dopaminergic system are increasingly

22 associated with neurodevelopmental disorders, including ASD. To further elucidate the role

23 of the dopaminergic system in ASD, we investigated the effects of embryonic exposure to

24 valproic acid (VPA) on the postnatal development of the mesencephalic DA system in the

25 domestic chick. We found that VPA affected the rostro-caudal distribution of DA neurons, without changing the expression levels of several dopaminergic markers in the mesencephalon. We also investigated a potential consequence of this altered DA neuronal distribution in the septum, a social brain area previously associated to social behaviour in several vertebrate species, describing alterations in the expression of genes linked to DA neurotransmission. These findings support the emerging hypothesis of a role of DA dysfunction in ASD pathogenesis. Together with previous studies showing impairments of early social orienting behaviour, these data also support the use of the domestic chick model to investigate the neurobiological mechanisms involved in early ASD symptoms.

\section{Introduction}

Autism spectrum disorder (ASD) comprise a group of neurodevelopmental conditions strongly characterized by impairments in sociability and social communication. In recent years, the role of the dopaminergic system in the regulation of social behaviour is being progressively outlined (Gunaydin and Deisseroth, 2014; Yamaguchi et al., 2017; Silva et al., 2020), often in association to motivational and reward mechanisms (Bariselli et al., 2018), and dysfunctions of the dopaminergic system in neurodevelopmental disorders and ASD has also been described (Scott-Van Zeeland et al., 2010; Supekar et al., 2018; Zürcher et al., 2021).

Approximately $75 \%$ of the total number of dopaminergic (DA) cells in the brain are located in the ventral part of the mesencephalon, giving rise to two major ascending DA systems that project to specific brain areas (Björklund and Dunnett, 2007). DA neurons in the ventral tegmental area (VTA) of the mesencephalon mainly project to the ventral striatum, the septum, the amygdala and the medial prefrontal cortex and constitute the mesocorticolimbic (or mesolimbic) pathway. Neurons of this pathway belong to the reward system and are 
49 thought to play a major role in controlling social reward, social learning and affiliative

50 behaviour. On the other hand, neurons originating in the substantia nigra (SN) and

51 innervating the dorsal striatum form the mesostriatal pathway, mainly involved in motor

52 control. Despite the distinct categorization, neurons of the SN and the VTA have a complex

53 organization and are intertwined in the mesencephalon, having also partially overlapping

54 projections and sharing similar functions (Wise, 2009; llango et al., 2014). Furthermore,

55 both DA mesencephalic nuclei are also blended with other type of neurons, as for example

56 GABA and glutamate releasing neurons (Morales and Margolis, 2017) that contribute to

57 their functional features (Bourdy et al., 2014).

In humans, the activation of the mesocorticolimbic pathway has been associated to processing of social stimuli (Spreckelmeyer et al., 2009), while a reduced activation of the 60 same pathway has been described in children and adults with ASD (Scott-Van Zeeland et al., 2010; Supekar et al., 2018). Pharmacological studies in mice have shown that administration of DA mimetic and antagonizing drugs can modulate social interaction bidirectionally. In a seminal work Gunaydin and coworkers (Gunaydin et al., 2014) demonstrated a causal link between activation of VTA DA neurons and social interaction in mice using optogenetic tools. In addition, chemogenetic inhibition of VTA neurons projecting to Nucleus Accumbens (NAc) in mice has been associated to reduced social novelty seeking (Bariselli et al., 2018) and the molecular bases of such mechanisms in neurodevelopmental disorders are starting to be elucidated (Bariselli et al., 2016, 2018). Recent studies have also highlighted the role of the lateral septum, a limbic structure mainly innervated by the VTA DA neurons, in sociability and social novelty seeking in mice (Mesic et al., 2015), emphasizing the role of DA neurotransmission in the regulation of social behaviour (Hostetler et al., 2017; Shin et al., 2018). Overall, it appears that activation of mesolimbic system, connecting the VTA to NAc and to the LS, have a prosocial effect on pair and maternal bonding, social stimuli processing as well as on social interaction.

In this study, we harnessed the evolutionary conserved nature of the DA neuromodulatory system to investigate in domestic chicks the neurobiological mechanisms affected by VPA and potentially linked to the social behavioural deficits underlying ASD. VPA is an anticonvulsant known to interfere with development of the social brain, whose prenatal exposure is associated in humans with neural tube malformations, reduced cognitive 
function and an increased risk for developing ASD (Christensen et al., 2013). VPA embryonic exposure has been extensively used to model ASD core symptoms in diverse animal species (see for a review Bambini-Junior et al., 2014) including the domestic chick, where it induces alterations of several aspects of social behaviour (Nishigori et al., 2013; Sgadò et al., 2018; Lorenzi et al., 2019; Zachar et al., 2019; Adiletta et al., 2021). Here we examined the anatomical and molecular layout of the mesencephalic DA system in domestic chicks exposed to VPA during embryonic development. We found that VPA affected the rostro-caudal distribution of DA neurons, without changing the gene expression levels of several dopaminergic markers in the mesencephalon. We also investigated a potential consequence of this altered DA neuronal distribution in the septum, a social brain area previously associated to social behaviour in several vertebrate species (Lorenzi et al., 2017; Mayer et al., 2017; Clemens et al., 2020) and we observed alterations in the expression of genes linked to DA neurotransmission.

\section{Material and Methods}

Ethical approval. All experiments were conducted according to the current Italian and

95 European Community laws for the ethical treatment of animals. The experimental procedures were approved by the Ethical Committee of the University of Trento and licensed by the Italian Health Ministry (permit number 986/2016-PR).

98 Embryo injections. Fertilized eggs of domestic chicks (Gallus gallus), of the Ross 308

99 (Aviagen) strain, were obtained from a local commercial hatchery (Agricola Berica,

100 Montegalda (VI), Italy). Upon arrival the eggs were incubated in the dark at $37.5^{\circ} \mathrm{C}$ and $10160 \%$ relative humidity, with rocking. One week before the predicted date of hatching, on 102 embryonic day 14 (E14), fertilized eggs were selected by a light test and injected. Chick

103 embryo injection was performed according to previous reports (Nishigori et al., 2013, Sgadò 104 et al., 2018). Briefly, a small hole was made on the flat end of the egg and either VPA

105 (Sodium Valproate, Sigma Aldrich, 35 umoles) or vehicle (double distilled injectable water;

106 CTRL group) were administered by dropping $200 \mu \mathrm{l}$ of solution into the air sac of each

107 fertilized egg. Eggs were assigned to the treatment groups randomly. After sealing the hole 108 with paper tape, eggs were placed back in the incubator until E18, when they were 109 prepared for hatching by incubation at $37.7^{\circ} \mathrm{C}$, with $72 \%$ humidity, and in complete 110 darkness. The day of hatching was considered post-hatching day 0 (P0). 
111 Immunohistochemistry. After two days of dark incubation, P2 chicks were overdosed by an

112 intramuscular injection of $0.05-\mathrm{ml}$ per $10 \mathrm{~g}$ of body weight of Ketamine/Xylazine Solution

113 (1:1 Ketamine $10 \mathrm{mg} / \mathrm{ml}+$ Xylazine $2 \mathrm{mg} / \mathrm{ml})$. After 5 minutes chicks were transcardially

114 perfused with phosphate-buffered saline (PBS) and ice-cold paraformaldehyde (4\% PFA in

115 PBS). Before removing the brain from the skulls, a coronal plane cut was performed using a

116 stereotaxic apparatus (Kuenzel and Masson, 1988) to ensure correct orientation following

117 the stereotaxic coordinates $\left(45^{\circ}\right)$ for coronal sectioning. Brains were then embedded in $7 \%$

118 bovine gelatine in PBS (4.2 g Bovine gelatine $+60 \mathrm{ml} \mathrm{PBS}$ at $40^{\circ} \mathrm{C}$ ), using the plane cut to

119 position the brain on the coronal plane. After cooling, the brains were post-fixed and

120 cryopreserved in 4\% PFA/PBS/20\% sucrose for approximately 6 hours at room

121 temperature, and then transferred to 30\% Sucrose/0.4\%PFA/PBS for further $72 \mathrm{~h}$. Brains

122 were then frozen in dry ice for 30 min before sectioning the entire brain in $60 \mu \mathrm{m}$ coronal

123 serial sections. For free-floating immunostaining, sections were washed 3 times in PBST

$124(0,005 \%$ Triton/PBS) between each of the following steps. After incubation in $0.3 \%$

$125 \mathrm{H}_{2} \mathrm{O}_{2} / \mathrm{PBS}$ for $20 \mathrm{~min}$, the sections were treated for 30 min with blocking solution (3\%

126 normal goat serum in PBS). Primary antibody reaction was carried out for 10 days at $4^{\circ} \mathrm{C}$ in

127 blocking solution (1:1000 mouse monoclonal Tyrosine Hydroxylase antibody; T2928,

128 Sigma-Aldrich). Sections were then incubated in biotinylated goat-anti-mouse antibody for

12924 hours at $4{ }^{\circ} \mathrm{C}$ in blocking solution (1:200, BA-1000, Vector Laboratories). Color reaction

130 was performed using the Vectastain Elite ABC Kit (PK-6100, Vector Laboratories) and the

131 DAB kit (SK-4600, Vector Laboratories) following the manufacturer instructions.

132 Cell counts Counts of TH positive cells in the substantia nigra and ventral tegmental area

133 were performed on 8 sets of serial sections per animal, sampled at $300 \mu m$ intervals $(n=3$

134 CTRL and 3 VPA chicks). Counting of TH immunoreactive cells was performed blind to the

135 experimental condition using ZEN imaging software (Zeiss, Germany). All the sections were

136 aligned on the rostral to caudal axis using Plate 34 of the chick brain atlas as reference

137 (Puelles et al., 2007) and a rectangle of $150 \times 250$ pixels was placed over the samples and

$138 \mathrm{TH}$-positive cells within this area were manually counted. Cell densities were separately

139 counted in the SN and the VTA as number of cells/area. For each analyzed brain slice, the

140 sampling field was moved randomly through the area of interest at least four times for each

141 dopaminergic subgroup. 
142 Tissue dissection. For microdissection of the SN and the VTA neurons, P2 chicks reared in

143 complete darkness were euthanized via carbon dioxide gaseous asphyxiation, their brain

144 extracted and then fast-frozen in dry-ice-cold isopentane solution. $100 \mu \mathrm{m}$ coronal sections

145 were cut using a Leica CM1850 UV Cryostat at $-15^{\circ} \mathrm{C}$, and stored at $-20^{\circ} \mathrm{C}$. To better

146 localize the targeted areas, sections were stained for 15 minutes with a $0.01 \%$ cresyl violet

147 solution dissolved in 100\% ethanol, and then progressively dehydrated in 75\%, 90\% and

148 100\% ethanol (1 minute/each). All solutions were prepared fresh and filter-sterilized to avoid

149 RNases contaminations. Substantia nigra and ventral tegmental area regions were finally

150 dissected out using a 20G needle and immediately processed for total RNA extraction. For

151 dissections of the septum, P2 chicks reared in darkness were euthanized by carbon dioxide

152 gaseous asphyxiation, the brain extracted, and the area of interest directly dissected.

153 Briefly, two coronal cuts were performed approximately $2 \mathrm{~mm}$ and $4 \mathrm{~mm}$ anterior to the

154 bregma to isolate the septum in the anterior-posterior axis according to (Puelles et al.,

155 2007) and the septum was carefully removed with forceps, fresh frozen in dry ice and

156 immediately processed for total RNA extraction.

157 Total RNA Extraction. Total RNA extraction from the septum was performed using the

158 RNeasy Mini Kit (QIAGEN), while RNA from SN and VTA samples was extracted using the

159 PicoPure ${ }^{T M}$ RNA Isolation Kit (Applied Biosystems ${ }^{T M}$, Thermo Fisher Scientific). Both

160 extractions were performed according to the manufacturers' instructions. Reverse

161 transcription for both types of extracted materials was performed with the SuperScript ${ }^{\mathrm{TM}}$

162 VILO ${ }^{\text {TM }}$ cDNA Synthesis Kit (Invitrogen, Thermo Fisher Scientific; Monza, Italy), following

163 manufacturer's instructions.

164 Reverse Transcription-Quantitative Polymerase Chain Reaction (RT-qPCR).RT-qPCR was

165 carried out with PowerUp ${ }^{\mathrm{TM}}$ SYBR ${ }^{\mathrm{TM}}$ Green Master Mix (2x) (Thermo Fisher Scientific;

166 Monza, Italy) for the septum and with SsoAdvanced ${ }^{\mathrm{TM}}$ Universal SYBR ${ }^{\circledR}$ Green Supermix

167 (Bio-Rad, Milan, Italy) for mesencephalic samples. Both reactions were performed using a

168 CFX96 ${ }^{\text {TM }}$ Real-Time System (Bio-Rad, Milan, Italy). Commercially synthesized primers

169 (Merck Life Science Srl, Milan, Italy) used in this work are listed in Table 1. Quantitation

170 cycles $(\mathrm{Cq})$ values were calculated using the second derivative maximum method. Data

171 were normalized on the expression of TBP (TATA-Box Binding Protein) and HMBS 
172 (Hydroxymethylbilane Synthase) reference genes using the DeltaCt (dCt) method (Pfaffl, 173 2001).

174 Table 1. Primers used for RT-qPCR

\begin{tabular}{|c|c|c|c|c|c|}
\hline Gene name & & Primer Sequence & Gene name & & Primer Sequence \\
\hline DRD1 & $\begin{array}{l}\text { Forward } \\
\text { Reverse }\end{array}$ & $\begin{array}{r}\text { CGTCTCATGTCTGCTATCTGTAAG } \\
\text { AAGAGTCCCTTTCCACAAGC }\end{array}$ & 5HTR2A & $\begin{array}{l}\text { Forward } \\
\text { Reverse }\end{array}$ & $\begin{array}{r}\text { ACCTCTGTGCCATCTCATTG } \\
\text { CCAAAGACAGGGATAGGCATG }\end{array}$ \\
\hline DRD2 & $\begin{array}{l}\text { Forward } \\
\text { Reverse }\end{array}$ & $\begin{array}{r}\text { GACAAATGCACTCATCCAGAAG } \\
\text { АСАCСАTCTCСАTTTCСАTCTC }\end{array}$ & TH & $\begin{array}{l}\text { Forward } \\
\text { Reverse }\end{array}$ & $\begin{array}{l}\text { CGAGACTTTGATCCTGATGCTG } \\
\text { GTATTTCACTGAGAAGGGCCTC }\end{array}$ \\
\hline GRIN2A & $\begin{array}{l}\text { Forward } \\
\text { Reverse }\end{array}$ & $\begin{array}{r}\text { ATACATCTTTGCCACTACGGG } \\
\text { AAATACCAGTCAGCCACAGG }\end{array}$ & TPH2 & $\begin{array}{l}\text { Forward } \\
\text { Reverse }\end{array}$ & $\begin{array}{l}\text { CAGTATGTACGACACGGCTC } \\
\text { TTCGTCAGATGCTCCCAATG }\end{array}$ \\
\hline GRIN2B & $\begin{array}{l}\text { Forward } \\
\text { Reverse }\end{array}$ & $\begin{array}{l}\text { CTTCATGGGTGTCTGCTCTG } \\
\text { GGATGTTGGAATGGGTGTTG }\end{array}$ & GABRA1 & $\begin{array}{l}\text { Forward } \\
\text { Reverse }\end{array}$ & $\begin{array}{r}\text { GAAGATGGCTCTCGACTGAAC } \\
\text { CCTCTTCAAGTGAAAATGTGTAGTC }\end{array}$ \\
\hline DARPP32 & $\begin{array}{l}\text { Forward } \\
\text { Reverse }\end{array}$ & $\begin{array}{l}\text { AGATCCAATTCTCAGTGCCG } \\
\text { ACTCGTCCTCTACATCTGGG }\end{array}$ & GABBR2 & $\begin{array}{l}\text { Forward } \\
\text { Reverse }\end{array}$ & $\begin{array}{r}\text { TTGGCTTGGGATTGTCTACG } \\
\text { CTCATTCCGATGTATTTGCTGTC }\end{array}$ \\
\hline $5 \mathrm{HTT}$ & $\begin{array}{l}\text { Forward } \\
\text { Reverse }\end{array}$ & $\begin{array}{r}\text { GCTACTGCATAGGAACCTCTTC } \\
\text { TTCTGTGGCTGTTTCTGGAG }\end{array}$ & GAD1 & $\begin{array}{l}\text { Forward } \\
\text { Reverse }\end{array}$ & $\begin{array}{r}\text { ATCCACCGCTAACACCAAC } \\
\text { CGCCATCTTTATTCGACCATCC }\end{array}$ \\
\hline CREB1 & $\begin{array}{l}\text { Forward } \\
\text { Reverse }\end{array}$ & $\begin{array}{l}\text { CTCCAGACGTTGACTATGACC } \\
\text { AGGTCTGTACATCTCCTGAGG }\end{array}$ & EN1 & $\begin{array}{l}\text { Forward } \\
\text { Reverse }\end{array}$ & $\begin{array}{r}\text { CTCAACGAGTCCCAGATCAAG } \\
\text { TCTTTGTCCTGCACCGTG }\end{array}$ \\
\hline OXTR & $\begin{array}{l}\text { Forward } \\
\text { Reverse }\end{array}$ & $\begin{array}{l}\text { TGTGCTGGACGCCCTTCT } \\
\text { TCCTGCGGAGCGTTGGT }\end{array}$ & TBP & $\begin{array}{l}\text { Forward } \\
\text { Reverse }\end{array}$ & $\begin{array}{r}\text { CTTCGTGCCCGAAATGCT } \\
\text { GCGCAGTAGTACGTGGTTCTCTT }\end{array}$ \\
\hline 5HTR1A & $\begin{array}{l}\text { Forward } \\
\text { Reverse }\end{array}$ & $\begin{array}{l}\text { ATTATGGGCACCTTCATCCTC } \\
\text { GCACTTACTGTCACAAAAGGG }\end{array}$ & HMBS & $\begin{array}{l}\text { Forward } \\
\text { Reverse }\end{array}$ & $\begin{array}{l}\text { GGTTGAGATGCTCCGTGAGTTT } \\
\text { GGCTCTTCTCCCCAATCTTAGAA }\end{array}$ \\
\hline
\end{tabular}

176 Statistical analysis. Statistical evaluation of the effect of treatment on the distribution and

177 number of DA neurons of the different subgroups and of the log2 gene expression levels

178 (dCt) was assessed using mixed-effect models using the nlme package in $\mathrm{R}$ (https://cran.r-

179 project.org/web/packages/nlme/index.html). For Tukey pairwise comparison tests, we used

180 the emmeans package in R (https://cran.r-project.org/web/packages/emmeans/index.html).

\section{$181 \quad$ Results}

182 Previous studies demonstrated a remarkable effect of exposure to VPA during embryonic

183 development on the dopaminergic system (Schiavi et al., 2019; Messina et al., 2020; Ádám

184 et al., 2020; Román et al., 2021). To evaluate the effect of VPA on the dopaminergic system 
185 of domestic chicks, we performed immunohistochemical analysis and quantification of DA

186 cell number in the SN and the VTA of VPA- and vehicle injected chicks, 48 hrs after

187 hatching (P2). Brain sections from VPA- and vehicle-injected domestic chicks were

188 immunolabeled for Tyrosine Hydroxylase (TH), the rate limiting enzyme for DA synthesis.

189 Representative images of TH immunohistochemically labelled cells in the SN and VTA are

190 shown in Fig1 A. TH-positive cells where then counted and DA cell densities (cells/area,

191 see Methods) were quantified in each of the serial sections encompassing the

192 mesencephalon, separating neurons of the SN from those of the VTA, and outlining their

193 respective rostro-caudal distribution. To assess the effect of treatment (VPA and CTRL), DA

194 group (SN and VTA) and rostro-caudal distribution (a set of 8 sections, from the most rostral

195 to the most caudal) on DA density, we used a linear mixed model (LMM), considering

196 treatment, group and section as fixed factors. We compared a model with random-

197 intercepts-only to one with random slope and intercepts, without covariance between

198 intercepts and slope, and found that the second model fitted the data significantly better.

199 We found that the overall density of DA cells was significantly different between the two DA

200 groups $\left(S N\right.$ vs VTA: $\left.F_{(1,60)}=40.2371, p<0.0001\right)$ and in the different rostro-caudal

201 positions (sections: $F_{(7,60)}=10.5726$, $p<0.0001$ ) but no significant main effect of treatment

202 was found in the overall number of DA cells (Fig. 1B; mean cells/area in substantia nigra

203 CTRL 5.574 [95\% C.I. 4.232 - 6.915], VPA 5.574 [95\% C.I. 2.451 - 8.696] in ventral

204 tegmental area CTRL 9.302 [95\% C.I. 2.354 - 16.250], VPA 9.229 [95\% C.I. 8.064 -

205 10.394; treatment: $\left.F_{(1,4)}=0.7096, p=0.4470\right)$. We also observed a significant interaction

206 between treatment and section (treatment ${ }^{*}$ section: $F_{(7,60)}=4.2912, p=0.0007$ ), indicating

207 an effect of treatment on the rostro-caudal distribution of DA neurons. More interestingly, a

208 triple interaction between treatment, section and group was observed

209 (treatment ${ }^{*}$ section*group: $F_{(7,60)}=2.2929, p=0.0387$ ), suggesting that the effect of

210 treatment on the rostro-caudal distribution was different in the two DA subgroups (Fig. 1C

211 and D). No other significant interactions were found between the other factors

212 (treatment ${ }^{\star}$ group: $F_{(1,60)}=0.0039, p=0.9503$; section*group: $F_{(7,60)}=0.50067, p=0.4812$ ).

213 Given the differences on the overall density of DA cells in the subgroups, we analysed VTA

214 and SN distribution separately. The pairwise comparison of the cell densities in VPA and

215 vehicle-treated domestic chicks in each section of the separate groups (Fig. 1C and D)

216 revealed a change in the distribution of the DA cell densities towards the posterior part of

217 the mesencephalon, and thus a caudal shift in the distribution of DA neurons in VPA- 
218 injected chick compared to controls, more prominent in the substantia nigra (Fig 1C; CTRL

219 vs VPA, section1 $\mathrm{t}_{(4)}=8.7974, \mathrm{p}=0.0009$; section $2 \mathrm{t}_{(4)}=3.1817, \mathrm{p}=0.0335$; section $3 \mathrm{t}_{(4)}=$ 220 2.4302, $\mathrm{p}=0.0720$; section $4 \mathrm{t}_{(4)}=1.9438, \mathrm{p}=0.1238$; section $5 \mathrm{t}_{(4)}=0.5757, \mathrm{p}=0.5957$;

221 section $6 \mathrm{t}_{(4)}=-3.5038, \mathrm{p}=0.0248$, section $7 \mathrm{t}_{(4)}=-3.7756, \mathrm{p}=0.0195$; section $8 \mathrm{t}_{(4)}=$ -

$2225.0059, p=0.0075$ ) than in the ventral tegmental area (Fig 1D; CTRL vs VPA, section1 $t_{(4)}=$ 223 3.3448, $\mathrm{p}=0.0287$; section $2 \mathrm{t}_{(4)}=1.0207, \mathrm{p}=0.3651$; section $3 \mathrm{t}_{(4)}=1.0597, \mathrm{p}=0.3490$;

224 section $4 t_{(4)}=-1.6494, p=0.1744$; section $5 t_{(4)}=-1.0495 p=0.3532$; section $6 t_{(4)}=$

$2250.4148, p=0.6996$, section $7 t_{(4)}=-0.2410 p=0.8214$; section $\left.8 t_{(4)}=-3.8945 p=0.0176\right)$.

226 Overall, our statistical analysis indicated a significant effect of VPA injection in the second

227 embryonic week on the development of the mesencephalic dopaminergic neurons

228 detectable at P2 as an alteration of the rostro-caudal distribution of DA cells.

229 To assess the molecular changes induced by VPA on the dopaminergic system at P2, we

230 micro-dissected DA neurons of the SN and VTA (Fig. 2A; $n=6$ animal per treatment group,

231 two independent experiments) in the entire rostro-caudal distribution, and measured the

232 expression levels of genes involved in development (En1, TH, TPH2, Gad1) and

233 neurotransmission (DRD1, DRD2, 5HTR1A, 5HTR2A, GABRA1, GABBR2). To assess the

234 effect of treatment, DA group and transcripts, we used a linear mixed model, considering

235 treatment, group and transcript as fixed factors and the experimental unit (experiment) as

236 random factor. We compared a model with random-intercepts-only to one with random

237 slopes and intercepts, and found that the random slopes and intercepts model fitted the

238 data significantly better. The statistical analysis indicated a significant difference in the

239 expression levels between the transcripts analysed in the two dopaminergic subgroups

240 (transcripts: $F_{(8,152)}=406.6968, p<0.0001$; group: $F_{(1,152)}=3.7786, p=0.0538$;

241 gene*group: $\left.F_{(8,152)}=42.0532, p<0.0001\right)$, however we could not detect any significant

242 effect of VPA exposure at E14 on the expression of the genes at P2 (treatment: $F_{(1,10)}=$

$243 \quad 0.0372, p=0.8509$; treatment ${ }^{*}$ gene: $F_{(8,152)}=0.2526, p=0.9795$; treatment ${ }^{*}$ group: $F_{(1,152)}=$

$2440.1096, p=0.7410$; treatment ${ }^{*}$ gene* group: $\left.F_{(8,152)}=0.7626, p=0.6362\right)$. Independent on

245 the treatment, some of the genes showed differences in expression levels between the two

246 dopaminergic subgroups as indicated by the Tukey pairwise comparison for the transcripts

247 in each DA subgroup (Fig. 2B; SN vs VTA). DRD2 $\left(\mathrm{t}_{(152)}=4.8848, \mathrm{p}<0.0001\right), \mathrm{TH}\left(\mathrm{t}_{(152)}=\right.$

248 6.2522, $\mathrm{p}<0.0001)$, GAD1 $\left(\mathrm{t}_{(152)}=4.4819, \mathrm{p}<0.0001\right)$ and GABRA1 $\left(\mathrm{t}_{(152)}=2.8885, \mathrm{p}=\right.$ 
0.0044) show increased levels in the SN compared to the VTA, while TPH2 $\left(\mathrm{t}_{(152)}=-\right.$ 15.5918, $p<0.0001$ ) was expressed at higher levels in the VTA.

251

We then examined gene expression changes in the septum, a brain area highly innervated by dopaminergic input coming from the SN and the VTA (Montagnese et al., 2008) and involved in social behaviour (Hostetler et al., 2017; Shin et al., 2018), that has been shown to activate in domestic chicks in response to exposure to conspecifics (Lorenzi et al., 2017; Mayer et al., 2017). We assessed the expression of gene involved in neurotransmission (Fig. 3; $n=8$ animals per group, four independent experiments), like the dopamine receptors (DRD1 and DRD2) together with the DA responsive protein DARPP32, the serotonin receptors (5HTR1A and 5HTR2A) with the serotonin transporter 5HTT and genes involved in synaptic plasticity coding for the NMDA subunits (GRIN2A and GRIN2B), and CREB1. We also measured the expression levels of the mesotocin receptor (OXTR) the avian homolog of oxytocin, since the lateral part of the septum is known to receive consistent oxytocinergic innervation (Loveland et al., 2019; Horiai et al., 2020). We again used linear mixed models to evaluate the effect of treatment (CTRL vs VPA), DA subgroup (SN vs VPA) and transcripts, and found that the best fitting model was a random slopes and intercepts model with the same parameters used for analysis of gene expression in the mesencephalon. We found a significant main effect of treatment $\left(F_{(1,138)}=7.7599, p=\right.$ $0.0061)$ and a significant differences in the levels of expression of the transcripts $\left(\mathrm{F}_{(9,138)}=\right.$ 39.1627, $\mathrm{p}<0.0001)$. We also observed a significant interaction between treatment and transcript $\left(\mathrm{F}_{(9,138)}=5.1513, \mathrm{p}<0.0001\right)$, indicating an effect of treatment on some of the transcripts. The Tukey pairwise comparison indicated that expression of DRD1 $\left(\mathrm{t}_{(138)}=\right.$ 2.0741, $\mathrm{p}=0.0399)$, DARPP32 $\left(\mathrm{t}_{(138)}=5.6749, \mathrm{p}<0.0001\right)$, and GRIN2A $\left(\mathrm{t}_{(138)}=2.9705, \mathrm{p}\right.$

$272=0.0035)$ was decreased in VPA-injected chicks (Fig. 3), while 5HTT $\left(\mathrm{t}_{(138)}=-2.0297, \mathrm{p}=\right.$ 0.0443) expression was increased by the treatment, and expression of the other transcripts did not change $\left(5\right.$ HTR1A: $t_{(138)}=1.4917, p=0.1381 ; 5 H T R 2 A: t_{(138)}=-0.5621, p=0.5749$; CREB1: $\mathrm{t}_{(138)}=0.7844, \mathrm{p}=0.4342 ;$ DRD2: $\mathrm{t}_{(138)}=1.5030, \mathrm{p}=0.1351 ;$ GRIN2B: $\mathrm{t}_{(138)}=$ 1.6429, $\mathrm{p}=0.1027$; OXTR: $\left.\mathrm{t}_{(138)}=-0.2558, \mathrm{p}=0.7985\right)$.

\section{Discussion}


areas and are already mature at birth, innervating the neonatal brain. Thus, they represent an ideal target to modulate complex cognitive abilities originating in early development, such as social orienting behaviour. Notably, the dopaminergic system has been shown to influence key aspects of social and affiliative behaviours in humans and other vertebrates, and to cooperate in modulating key components of the social brain network (Gunaydin and Deisseroth, 2014). Moreover, accumulating evidence point to an involvement of DA neurotransmission in atypical social development in children with ASD (Scott-Van Zeeland et al., 2010; Supekar et al., 2018; Zürcher et al., 2021). In the present study, we investigated developmental dysregulations of the DA system in a VPA model of ASD implemented in domestic chicks, that could potentially contribute to the behavioural deficits observed in the chicks' spontaneous responses to social stimuli, including altered response to faces (Adiletta et al., 2021). More specifically, we analyzed the number, distribution, and the developmental gene expression of DA neurons in the postnatal mesecephalon of domestic chicks embryonically exposed to VPA, and then assessed gene expression changes in the septum, a region of the social brain network highly innervated by DA terminals (Lindvall and Stenevi, 1978; Gaspar et al., 1985) and involved in sociability and social novelty seeking (Mesic et al., 2015).

Consistent with our hypothesis of an effect of VPA on the DA system, when sampling the rostro-caudal portions of the mesencephalon, we observed a caudal shift in the distribution of mesencephalic DA population at P2, nine days after embryonic exposure to VPA.

300 Neuroanatomical alterations of mesencephalic TH population was already described in mice exposed to VPA by Ádám et al., (2020), however without analyses on the distribution of the DA population. Moreover, differently from the work of Ádám and colleagues (2020), here we found no difference in the total number of DA neurons (measured as number of cells/area) between the two treatments groups, suggesting the preservation of the overall profile of the DA population in our model, as indicated also by our gene expression analysis in the mesencephalon. Interestingly, VPA exposure was performed at E14, long after chick's dopaminergic proliferation and differentiation events have terminated (Andersson et al., 2006), deeming unlikely a direct influence of VPA on neurogenesis or differentiation of new DA neurons. To investigate the potential consequences of the observed rostro-caudal shift

310 in the distribution of the mesencephalic DA neurons, we have also assessed changes in the 311 expression of genes involved in DA neurotransmission in one of the target regions of the 
312 mesocorticolimbic pathway, the septum. We found that DRD1, DARPP32 and GRIN2A

313 were downregulated upon VPA exposure, consistent with deficits in dopaminergic signaling

314 in this brain region. We also found that expression of the serotonin transporter (5HTT) was

315 increased in the septum, suggestive of alterations also in serotonergic neurotransmission.

316 Several studies and meta-analysis have confirmed an increase in 5HT in the blood

317 (hyperserotonemia) of autistic individuals, such that hyperserotonemia has become a

318 reliable biomarker for these disorders (see for a review Lam et al., 2006; and Gabriele et al.,

319 2014). Epidemiological and animal model studies have suggested that perinatal alterations

320 in $5 \mathrm{HT}$, either above or below typical levels, may cause social behavioural deficits

321 resembling ASD (Garbarino et al., 2019).

322 Previous studies have reported alterations in the number of DA neurons or in DA

323 neurotransmission in several animal models of ASD (see for a recent review Kosillo and

324 Bateup, 2021). A reduction in the number of DA neurons in the SN (but not the VTA) was

325 found in adult mice lacking the Fmr1 gene (Fish et al., 2013). Further studies demonstrated

326 reduced striatal DA transmission and striatal DA re-uptake without significant changes in the

327 striatal tissue DA content in Fmr1 mutant mice (Fulks et al., 2010; Sørensen et al., 2015).

328 Alterations in DA-mediated responses have also been reported in the BTBR mice (Squillace

329 et al., 2014), a model for idiopathic autism, accompanied by decreased TH expression in

330 several DA innervated brain regions (Chao et al., 2020). Interestingly, intranasal dopamine

331 administration efficiently rescued the cognitive and social deficits of both BTBR and Fmr1

332 mutant ASD models (Chao et al., 2020), suggesting a causal role of DA deficiency in the

333 behavioural phenotype of the mice.

334 Notably, in our study, we investigated for the first time DA-related deficits on an ASD model

335 implemented in the domestic chicks. Differently from other common animal models, chicks

336 are precocial species able to independently behave soon after hatching (Versace and

337 Vallortigara, 2015), displaying remarkable early and spontaneous social responses, already

338 shown to have similar features to the social orienting behaviour observed in human

339 newborns (Di Giorgio et al., 2017). Domestic chicks also enable to study early

340 neurodevelopmental mechanisms, without the interference of sophisticated, divergent,

341 strategies of adaptive learning that emerge later. We thus believe that the domestic chick

342 represents an ideal candidate model to study the causal relationship between social 
343 orienting behaviour, emerging at early postnatal stages, and any underlying neurobiological

344 alterations mediated by VPA or any other genetic manipulation associated to ASD. Further

345 studies should thus investigate the potential causal relationship between DA signaling

346 alterations and the early social orienting deficits observed in VPA exposed chicks, including

347 impairments in face processing and affiliative behaviour.

\section{Ethics approval}

349 All experiments were conducted according to the current Italian and European Community 350 laws for the ethical treatment of animals. The experimental procedures were approved by

351 the Ethical Committee of the University of Trento and licensed by the Italian Health Ministry 352 (permit number 986/2016-PR).

\section{Conflict of Interest}

354 The authors declare that the research was conducted in the absence of any commercial or

355 financial relationships that could be construed as a potential conflict of interest.

\section{Author Contributions}

357 PS conceived and designed the experiments; AA and AP conducted the experiments; NT

358 provided technical support; PS and AA analyzed the data; AA and PS wrote the manuscript.

359 All authors read and approved the final manuscript.

\section{$360 \quad$ Funding}

361 This work was supported by the University of Trento (intramural funds to AA, NT and PS).

\section{Acknowledgments}

363 We thank Giorgio Vallortigara and Andrea Messina for their support. Dr. Tommaso Pecchia 364 for help with the experimental apparatus, Grazia Gambardella and Roberta Guidolin for 365 administrative help and Ciro Petrone for animal facility management.

\section{Data Availability Statement}

367 All data generated or analysed during this study are included in this published article (see 368 supplementary information). 
Figure Legends

370 Figure 1. Immunohistochemical analysis and quantification of cell densities. (A) Brain

371 sections from CTRL and VPA chicks immunolabeled for Tyrosine Hydroxylase (TH). (B)

372 Number of TH-positive DA neurons in both SN and VTA. TH-positive cells' count was

373 performed on 8 sets of serial sections per animal, sampled at $300 \mu \mathrm{m}$ intervals. Rostro-

374 caudal alignment of the brain sections was based on atlas reference (Plate 34 from Puelles

375 et al., 2007). (C) Substantia Nigra DA cell density measured in its rostro-caudal distribution.

376 (D) Ventral Tegmental Area DA cell density measured in its rostro-caudal distribution. * $p<$ $3770.05,{ }^{* * *} p<0.001$.

378 Figure 2. Gene expression levels in the SN and VTA. (A) Schematic representation of 379 the microdissected areas of interest (SN, Substantia Nigra; VTA, Ventral Tegmental Area) 380 from coronal sections (adapted from Plate 33 in Puelles et al., 2007). (B) Box and whisker

381 plot (median, min to max) of relative expression ( $d C t$, log2) values for each group. Changes

382 in expression of DRD1, DRD2, TH, TPH2, EN1, GABRA1, GABBR2, GAD1 and 5HTR2A in 383 both SN and VTA of VPA- and vehicle-injected chicks analyzed at P2. ${ }^{* *} p<0.01,{ }^{* * *} p<$ 3840.001.

385 Figure 3. Gene expression levels in the septum. Box and whisker plot (median, min to 386 max) of relative expression ( $\mathrm{dCt}$, log2) values for each group. Changes in expression of 387 DRD1, DRD2, DARPP32, 5HTT, GRIN2A, GRIN2B, CREB1, OXTR, 5HTR1A and 5HTR2A 388 in septum's samples collected from P2 chicks embryonically exposed to VPA. Box and 389 whiskers plot (median, min to max) of dCt values for each group. ${ }^{*} p<0.05$, ${ }^{\star * *} p<0.001$. 


\section{References}

392

393

394

395

396

397

398

399

400

401

402

403

404

405

406

407

408

409

410

411

412

413

414

415

416

417

418

419

420

421

422

423

424

425

426

427

428

Ádám, Á., Kemecsei, R., Company, V., Murcia-Ramón, R., Juarez, I., Gerecsei, L., et al. (2020). Gestational Exposure to Sodium Valproate Disrupts Fasciculation of the Mesotelencephalic Dopaminergic Tract, With a Selective Reduction of Dopaminergic Output From the Ventral Tegmental Area. Front Neuroanat 14, 29.

Adiletta, A., Pedrana, S., Rosa-Salva, O., and Sgadò, P. (2021). Spontaneous visual preference for face-like stimuli is impaired in newly-hatched domestic chicks exposed to valproic acid during embryogenesis. bioRxiv, 2021.04.12.436924.

Andersson, E., Tryggvason, U., Deng, Q., Friling, S., Alekseenko, Z., Robert, B., et al. (2006). Identification of intrinsic determinants of midbrain dopamine neurons. Cell 124, 393-405. doi:10.1016/j.cell.2005.10.037.

Bambini-Junior, V., Baronio, D., MacKenzie, J., Zanatta, G., Riesgo, R. dos S., and Gottfried, C. (2014). "Prenatal Exposure to Valproate in Animals and Autism," in Comprehensive Guide to Autism (New York, NY: Springer New York), 1779-1793.

Bariselli, S., Hörnberg, H., Prévost-Solié, C., Musardo, S., Hatstatt-Burklé, L., Scheiffele, P., et al. (2018). Role of VTA dopamine neurons and neuroligin 3 in sociability traits related to nonfamiliar conspecific interaction. Nature Communications 2018 9:1 9, 1-15. doi:10.1038/s41467-018-05382-3.

Bariselli, S., Tzanoulinou, S., Glangetas, C., Prévost-Solié, C., Pucci, L., Viguié, J., et al. (2016). SHANK3 controls maturation of social reward circuits in the VTA. Nat Neurosci 19, 926934.

Björklund, A., and Dunnett, S. (2007). Dopamine neuron systems in the brain: an update. Trends Neurosci 30, 194-202.

Bourdy, R., Sánchez-Catalán, M.-J., Kaufling, J., Balcita-Pedicino, J. J., Freund-Mercier, M.-J., Veinante, P., et al. (2014). Control of the nigrostriatal dopamine neuron activity and motor function by the tail of the ventral tegmental area. Neuropsychopharmacology 39, 2788-2798. doi:10.1038/npp.2014.129.

Chao, O. Y., Pathak, S. S., Zhang, H., Dunaway, N., Li, J.-S., Mattern, C., et al. (2020). Altered dopaminergic pathways and therapeutic effects of intranasal dopamine in two distinct mouse models of autism. Molecular Brain 13.

Christensen, J., Grønborg, T., Sørensen, M., Schendel, D., Parner, E., Pedersen, L., et al. (2013). Prenatal valproate exposure and risk of autism spectrum disorders and childhood autism. JAMA 309, 1696-1703.

Clemens, A. M., Wang, H., and Brecht, M. (2020). The lateral septum mediates kinship behavior in the rat. Nat Commun 11, 3161. doi:10.1038/s41467-020-16489-x.

Di Giorgio, E., Loveland, J. L., Mayer, U., Rosa-Salva, O., Versace, E., and Vallortigara, G. (2017). Filial responses as predisposed and learned preferences: Early attachment in chicks and babies. Behav Brain Res 325, 90-104. doi:10.1016/j.bbr.2016.09.018. 
Fish, E. W., Krouse, M. C., Stringfield, S. J., Diberto, J. F., Robinson, J. E., and Malanga, C. J. (2013). Changes in sensitivity of reward and motor behavior to dopaminergic, glutamatergic, and cholinergic drugs in a mouse model of fragile X syndrome. PLoS One 8, e77896. doi:10.1371/journal.pone.0077896.

Fulks, J. L., O’Bryhim, B. E., Wenzel, S. K., Fowler, S. C., Vorontsova, E., Pinkston, J. W., et al. (2010). Dopamine Release and Uptake Impairments and Behavioral Alterations Observed in Mice that Model Fragile X Mental Retardation Syndrome. ACS Chem Neurosci 1, 679-690. doi:10.1021/cn100032f.

Gabriele, S., Sacco, R., and Persico, A. M. (2014). Blood serotonin levels in autism spectrum disorder: a systematic review and meta-analysis. Eur Neuropsychopharmacol 24, 919-929. doi:10.1016/j.euroneuro.2014.02.004.

Garbarino, V. R., Gilman, T. L., Daws, L. C., and Gould, G. G. (2019). Extreme enhancement or depletion of serotonin transporter function and serotonin availability in autism spectrum disorder. Pharmacol Res 140, 85-99. doi:10.1016/j.phrs.2018.07.010.

Gaspar, P., Berger, B., Alvarez, C., Vigny, A., and Henry, J. P. (1985). Catecholaminergic innervation of the septal area in man: Immunocytochemical study using TH and DBH antibodies. Journal of Comparative Neurology 241, 12-33. doi:10.1002/cne.902410103.

Gunaydin, L. A., and Deisseroth, K. (2014). Dopaminergic Dynamics Contributing to Social Behavior. Cold Spring Harbor Symposia on Quantitative Biology 79, 221-227. doi:10.1101/SQB.2014.79.024711.

Gunaydin, L., Grosenick, L., Finkelstein, J., Kauvar, I., Fenno, L., Adhikari, A., et al. (2014). Natural neural projection dynamics underlying social behavior. Cell 157, 1535-1551.

Horiai, M., Otsuka, A., Hidema, S., Hiraoka, Y., Hayashi, R., Miyazaki, S., et al. (2020). Targeting oxytocin receptor (Oxtr)-expressing neurons in the lateral septum to restore social novelty in autism spectrum disorder mouse models. Sci Rep 10, 22173. doi:10.1038/s41598-020-791090.

Hostetler, C. M., Hinde, K., Maninger, N., Mendoza, S. P., Mason, W. A., Rowland, D. J., et al. (2017). Effects of pair bonding on dopamine D1 receptors in monogamous male titi monkeys (Callicebus cupreus). Am J Primatol 79, 1-9. doi:10.1002/ajp.22612.

Ilango, A., Kesner, A. J., Keller, K. L., Stuber, G. D., Bonci, A., and Ikemoto, S. (2014). Similar roles of substantia nigra and ventral tegmental dopamine neurons in reward and aversion. $J$ Neurosci 34, 817-822. doi:10.1523/JNEUROSCI.1703-13.2014.

Kosillo, P., and Bateup, H. (2021). Dopaminergic Dysregulation in Syndromic Autism Spectrum Disorders: Insights From Genetic Mouse Models. Front Neural Circuits 15, 700968.

Kuenzel, W. J., and Masson, M. (1988). A Stereotaxic Atlas of the Brain of the Chick (Gallus Domesticus). Johns Hopkins University Press.

Lam, K. S. L., Aman, M. G., and Arnold, L. E. (2006). Neurochemical correlates of autistic disorder: a review of the literature. Res Dev Disabil 27, 254-289. doi:10.1016/j.ridd.2005.03.003. 
Lindvall, O., and Stenevi, U. (1978). Dopamine and noradrenaline neurons projecting to the septal area in the rat. Cell Tissue Res 190, 383-407. doi:10.1007/BF00219554.

Lorenzi, E., Mayer, U., Rosa-Salva, O., and Vallortigara, G. (2017). Dynamic features of animate motion activate septal and preoptic areas in visually naïve chicks (Gallus gallus). Neuroscience 354, 54-68.

Lorenzi, E., Pross, A., Rosa-Salva, O., Versace, E., Sgadò, P., and Vallortigara, G. (2019). Embryonic Exposure to Valproic Acid Affects Social Predispositions for Dynamic Cues of Animate Motion in Newly-Hatched Chicks. Front Physiol 10, 501.

Loveland, J. L., Stewart, M. G., and Vallortigara, G. (2019). Effects of oxytocin-family peptides and substance $\mathrm{P}$ on locomotor activity and filial preferences in visually naïve chicks. Eur $J$ Neurosci 50, 3674-3687. doi:10.1111/ejn.14520.

Mayer, U., Rosa-Salva, O., and Vallortigara, G. (2017). First exposure to an alive conspecific activates septal and amygdaloid nuclei in visually-naïve domestic chicks (Gallus gallus). Behav Brain Res 317, 71-81.

Mesic, I., Guzman, Y. F., Guedea, A. L., Jovasevic, V., Corcoran, K. A., Leaderbrand, K., et al. (2015). Double Dissociation of the Roles of Metabotropic Glutamate Receptor 5 and Oxytocin Receptor in Discrete Social Behaviors. Neuropsychopharmacology 40, 2337-2346. doi:10.1038/npp.2015.81.

Messina, A., Boiti, A., Sovrano, V., and Sgadò, P. (2020). Micromolar Valproic Acid Doses Preserve Survival and Induce Molecular Alterations in Neurodevelopmental Genes in Two Strains of Zebrafish Larvae. Biomolecules 10.

Montagnese, C., Zachar, G., Bálint, E., and Csillag, A. (2008). Afferent connections of septal nuclei of the domestic chick (Gallus domesticus): a retrograde pathway tracing study. J Comp Neurol 511, 109-150.

Morales, M., and Margolis, E. (2017). Ventral tegmental area: cellular heterogeneity, connectivity and behaviour. Nat Rev Neurosci 18, 73-85.

Nishigori, H., Kagami, K., Takahashi, A., Tezuka, Y., Sanbe, A., and Nishigori, H. (2013). Impaired social behavior in chicks exposed to sodium valproate during the last week of embryogenesis. Psychopharmacology (Berl) 227, 393-402.

Pfaffl, M. W. (2001). A new mathematical model for relative quantification in real-time RT-PCR. Nucleic acids research 29, e45.

Puelles, L., Martinez-de-la-Torre, M., Martinez, S., Watson, C., and Paxinos, G. (2007). The Chick Brain in Stereotaxic Coordinates: An Atlas featuring Neuromeric Subdivisions and Mammalian Homologies. Elsevier Science.

Román, V., Adham, N., Foley, A., Hanratty, L., Farkas, B., Lendvai, B., et al. (2021). Cariprazine alleviates core behavioral deficits in the prenatal valproic acid exposure model of autism spectrum disorder. Psychopharmacology (Berl) 238, 2381-2392. 
504

505

506

507

508

509

510

511

512

513

514

515

516

517

518

519

520

521

522

523

524

525

526

527

528

529

530

531

532

533

534

535

536

537

538

539

540

Schiavi, S., Iezzi, D., Manduca, A., Leone, S., Melancia, F., Carbone, C., et al. (2019). RewardRelated Behavioral, Neurochemical and Electrophysiological Changes in a Rat Model of Autism Based on Prenatal Exposure to Valproic Acid. Front Cell Neurosci 13, 479.

Scott-Van Zeeland, A., Dapretto, M., Ghahremani, D., Poldrack, R., and Bookheimer, S. (2010). Reward processing in autism. Autism Res 3, 53-67.

Sgadò, P., Rosa-Salva, O., Versace, E., and Vallortigara, G. (2018). Embryonic Exposure to Valproic Acid Impairs Social Predispositions of Newly-Hatched Chicks. Sci Rep 8, 5919.

Shin, S., Pribiag, H., Lilascharoen, V., Knowland, D., Wang, X.-Y., and Lim, B. K. (2018). Drd3 Signaling in the Lateral Septum Mediates Early Life Stress-Induced Social Dysfunction. Neuron 97, 195-208.e6. doi:10.1016/j.neuron.2017.11.040.

Silva, P. A., Trigo, S., Marques, C. I., Cardoso, G. C., and Soares, M. C. (2020). Experimental evidence for a role of dopamine in avian personality traits. Journal of Experimental Biology 223. doi:10.1242/JEB.216499.

Sørensen, E. M., Bertelsen, F., Weikop, P., Skovborg, M. M., Banke, T., Drasbek, K. R., et al. (2015). Hyperactivity and lack of social discrimination in the adolescent Fmr1 knockout mouse. Behav Pharmacol 26, 733-740. doi:10.1097/FBP.0000000000000152.

Spreckelmeyer, K., Krach, S., Kohls, G., Rademacher, L., Irmak, A., Konrad, K., et al. (2009). Anticipation of monetary and social reward differently activates mesolimbic brain structures in men and women. Soc Cogn Affect Neurosci 4, 158-165.

Squillace, M., Dodero, L., Federici, M., Migliarini, S., Errico, F., Napolitano, F., et al. (2014). Dysfunctional dopaminergic neurotransmission in asocial BTBR mice. Transl Psychiatry 4, e427. doi:10.1038/tp.2014.69.

Supekar, K., Kochalka, J., Schaer, M., Wakeman, H., Qin, S., Padmanabhan, A., et al. (2018). Deficits in mesolimbic reward pathway underlie social interaction impairments in children with autism. Brain 141, 2795-2805.

Versace, E., and Vallortigara, G. (2015). Origins of Knowledge: Insights from Precocial Species. Front Behav Neurosci 9, 338. doi:10.3389/fnbeh.2015.00338.

Wise, R. (2009). Roles for nigrostriatal-not just mesocorticolimbic-dopamine in reward and addiction. Trends Neurosci 32, 517-524.

Yamaguchi, Y., Lee, Y.-A., Kato, A., Jas, E., and Goto, Y. (2017). The Roles of Dopamine D2 Receptor in the Social Hierarchy of Rodents and Primates. Scientific Reports 2017 7:1 7, 110. doi:10.1038/srep43348.

Zachar, G., Tóth, A., Gerecsei, L., Zsebők, S., Ádám, Á., and Csillag, A. (2019). Valproate Exposure in ovo Attenuates the Acquisition of Social Preferences of Young Post-hatch Domestic Chicks. Front Physiol 10, 881.

Zürcher, N. R., Walsh, E. C., Phillips, R. D., Cernasov, P. M., Tseng, C.-E. J., Dharanikota, A., et al. (2021). A simultaneous [11C]raclopride positron emission tomography and functional 
bioRxiv preprint doi: https://doi.org/10.1101/2021.11.08.467690; this version posted November 12, 2021. The copyright holder for this preprint (which was not certified by peer review) is the author/funder. All rights reserved. No reuse allowed without permission.

541

magnetic resonance imaging investigation of striatal dopamine binding in autism. Transl

542 Psychiatry 11, 1-11. doi:10.1038/s41398-020-01170-0.

543 
$A$

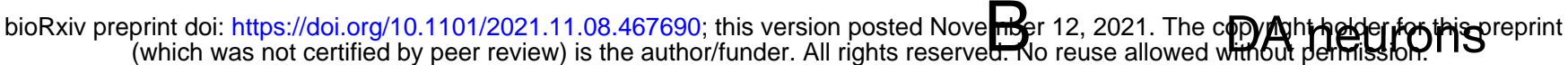
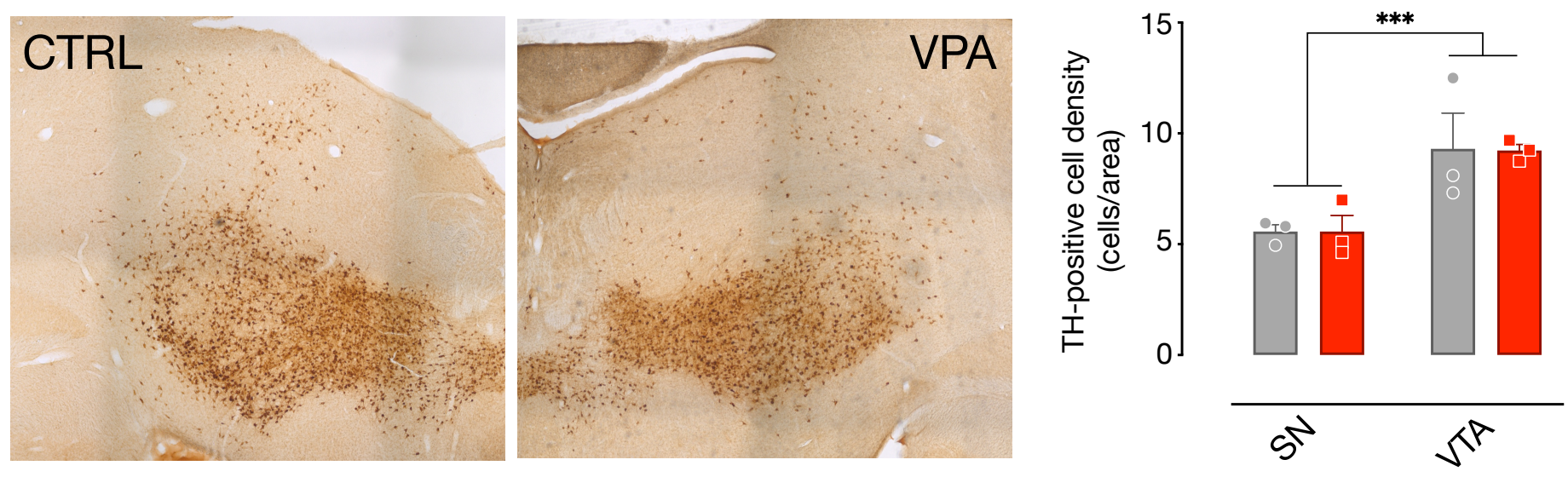

$\square$ CTRL $\square$ VPA

C

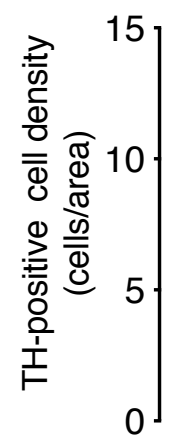

Substantia Nigra
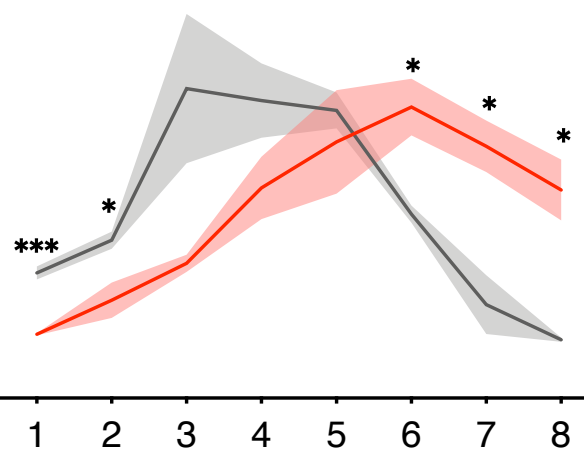

section (rostral to caudal)
D

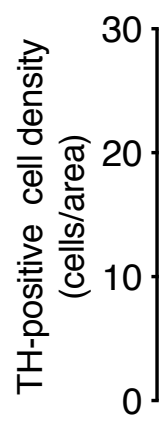

Ventral Tegmental Area

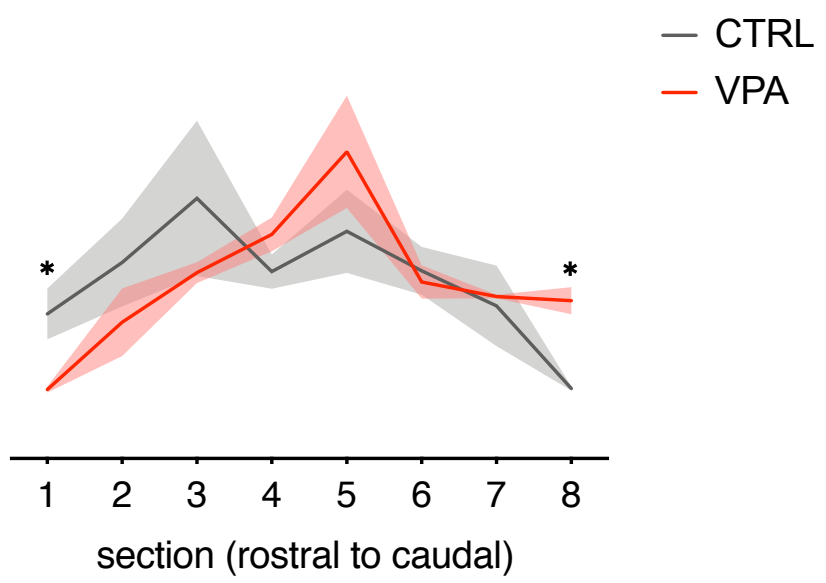




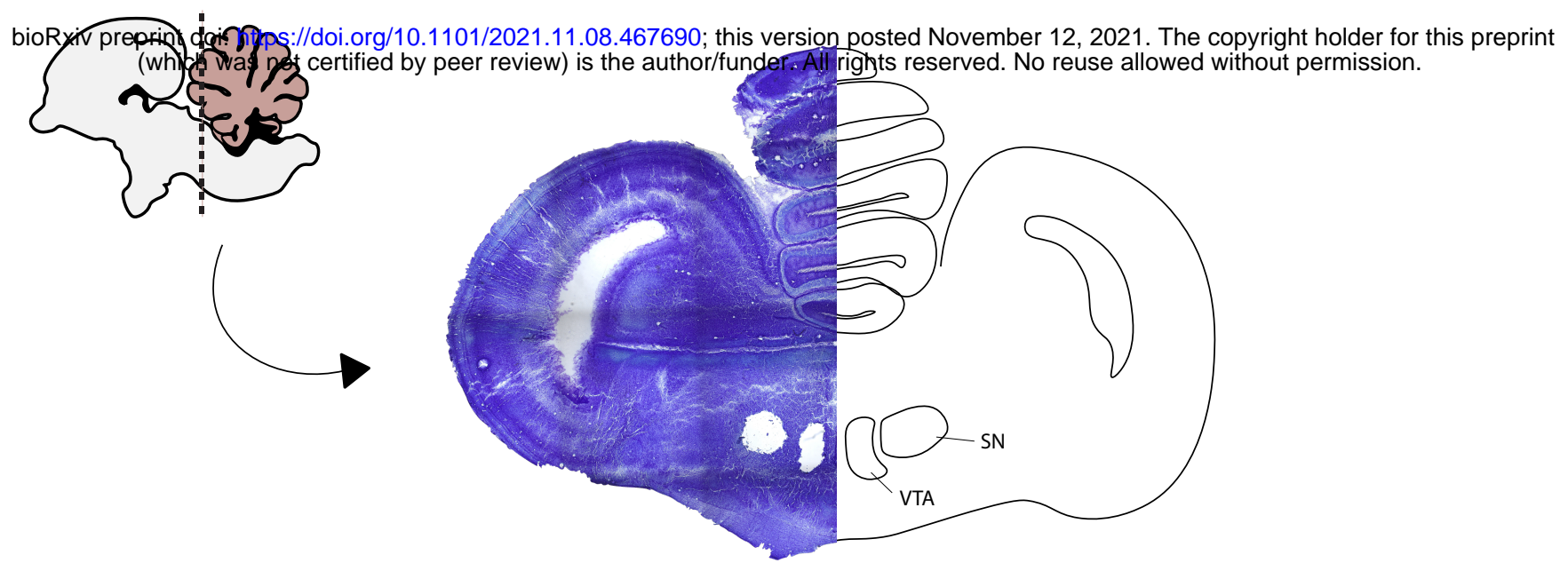

B

\section{Mesencephalic DA neurons}

$\square$ SN - CTRL $\square$ SN - VPA $\square$ VTA - CTRL $\square$ VTA - VPA
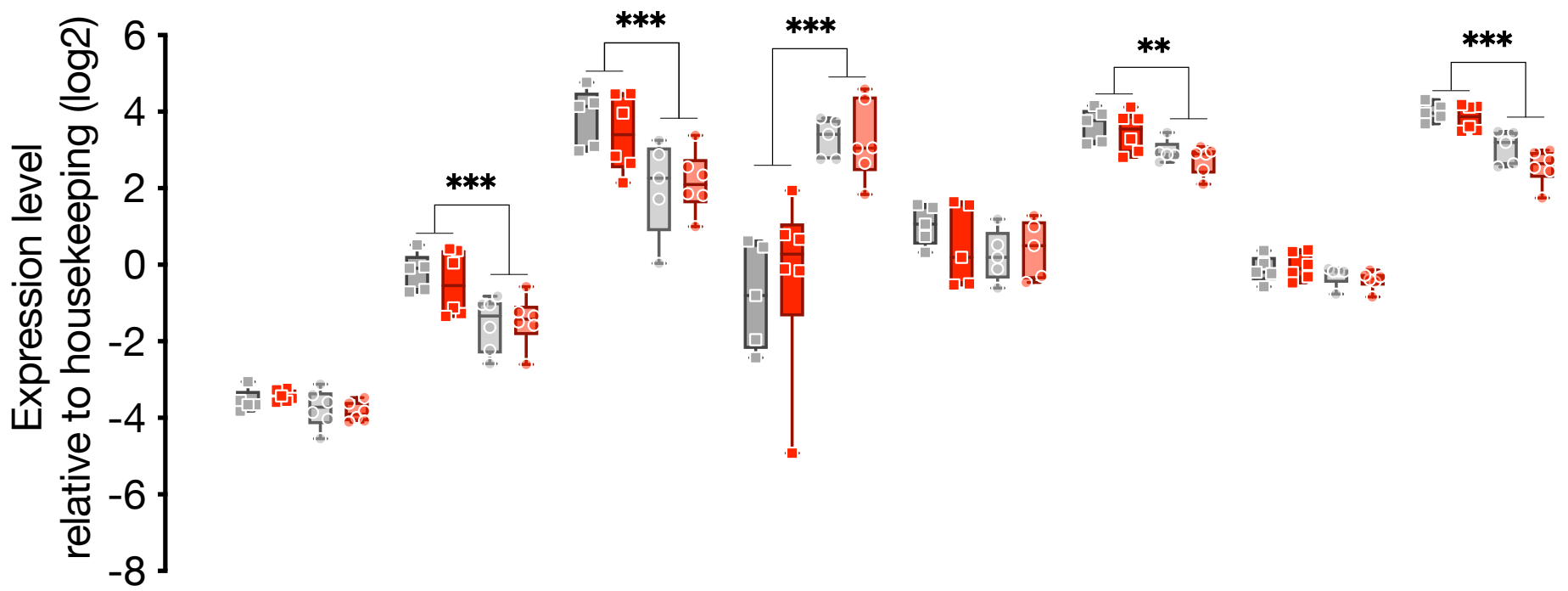

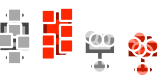<smiles></smiles><smiles>[C-]1C=[Ge]O1</smiles><smiles>C1=CC1</smiles><smiles>C1=[Ge]=C1</smiles><smiles>C1=CC=C1</smiles><smiles>C1=C=CC=C=1</smiles><smiles>O=C=[Os]</smiles><smiles>C1=C[C-][O+]=1</smiles><smiles>C1CCCCC1</smiles> 


\section{Septum}

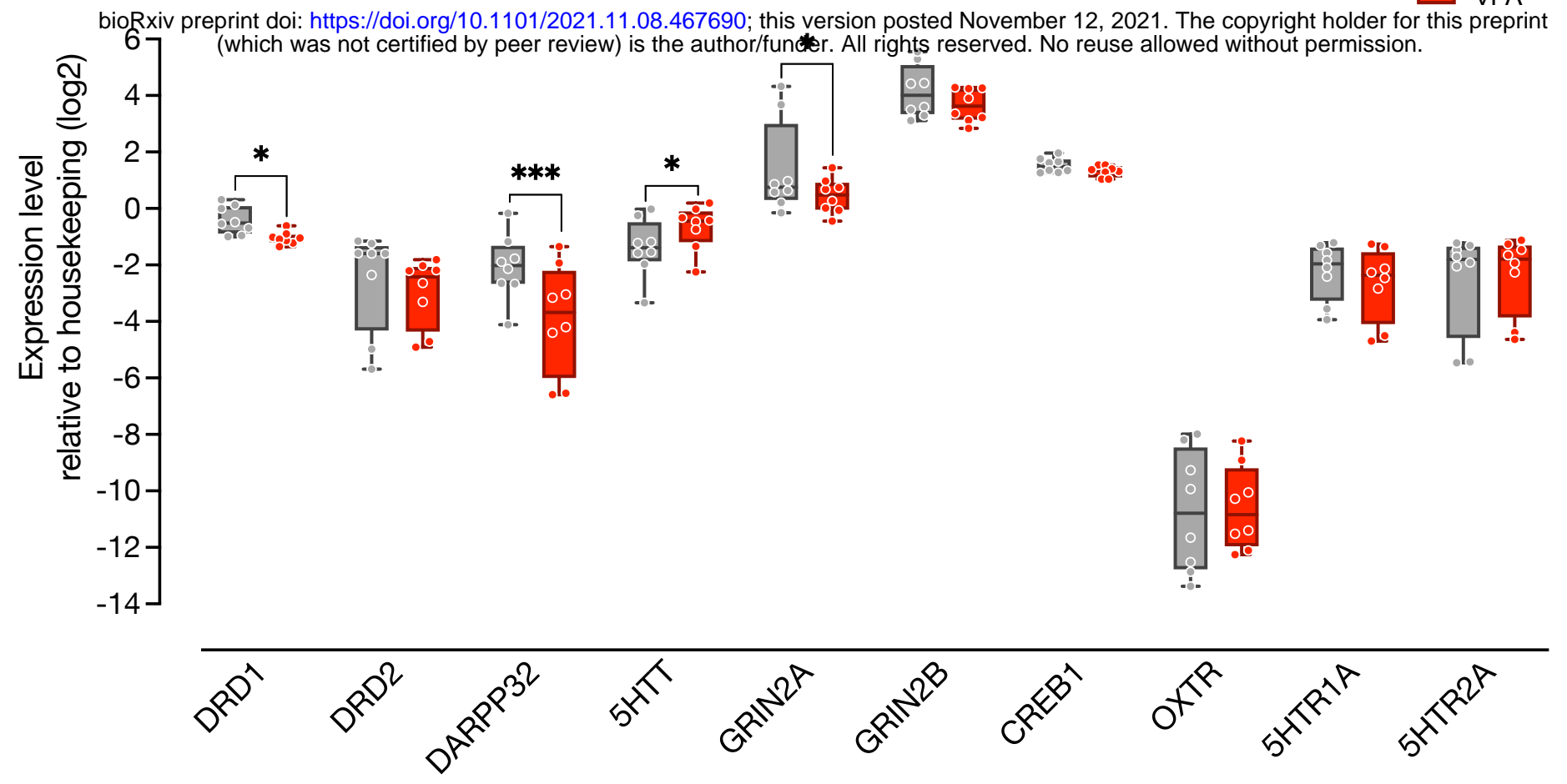

